\title{
Viewpoint
}

\section{Excited atoms spin out of equilibrium}

\author{
Igor Lesanovsky \\ School of Physics and Astronomy, University of Nottingham, Nottingham NG7 2RD, \\ United Kingdom
}

Published September 12, 2011

Excited cold atoms in Rydberg states behave similarly to certain spin systems, providing us with a versatile toolbox with which to study nonequilibrium phenomena.

Subject Areas: Atomic and Molecular Physics

A Viewpoint on:

Antiferromagnetic phase transition in a nonequilibrium lattice of Rydberg atoms

Tony E. Lee, H. Häffner, and M. C. Cross

Phys. Rev. A 84, 031402 (2011) - Published September 12, 2011

A promising approach towards probing matter far from equilibrium, a fundamental challenge in physics, calls for the usage of cold atoms. Gases of such atoms possess temperatures of a few microkelvin, or even lower, and can be almost perfectly isolated from the environment. Furthermore, the interaction strength, as well as their external trapping potential, can be controlled to an outstandingly high precision [1. They provide a platform not only for studying the dynamics of closed quantum systems but also for detailed exploration of the competition between coherent and dissipative dynamics within open systems with tailor-made properties [2].

In a paper in Physical Review A[3], Tony Lee at the California Institute of Technology, Pasadena, and colleagues theoretically investigate such an open quantum system. Their setting is a gas of alkali metal atoms confined to a regular lattice with exactly one atom per site - a setup that can be achieved experimentally with appropriately shaped laser beams or magnetic fields.

What makes the setting different from traditional experiments with cold atoms, and therefore particularly interesting, is the use of atoms in highly excited states - socalled Rydberg states [4. An alkali-metal atom, with its single active electron, shares many properties with the hydrogen atom. Excited states form a Rydberg series whose states can be labeled, just like in hydrogen, by the principal quantum number $n$. Interesting physics emerges in the presence of more than one Rydberg atom, as the large distance between the nucleus and the valence electron renders these atoms into electric dipoles. Depending on the particular Rydberg state, the interaction between two such atoms is then either determined by a van der Waals or a dipole-dipole potential. The authors consider the former potential, which is, in principle, also present between ground-state atoms. The striking difference, however, is that the interaction between atoms in Rydberg states is enhanced by a factor of up to $n^{11}$. For

DOI: $10.1103 /$ Physics.4.71

URL: http://link.aps.org/doi/10.1103/Physics .4.71 values of the principal quantum number typically used in experiments, $n=40 \ldots 80$, this means an increase of 10 orders of magnitude, i.e., the interaction affects even atoms that are separated by several micrometers. This is in contrast to the contact potential usually present between ground-state atoms. In the most extreme case, interaction-induced level shifts are so huge that a simultaneous excitation of two nearby atoms to Rydberg states is virtually impossible [for an illustration see Fig. 1(a)]. This so-called Rydberg blockade mechanism [5] lies behind a number of exciting phenomena that make Rydberg atoms useful for applications ranging from quantum information processing and quantum simulation to nonlinear quantum optics and ultracold chemistry.

A recent development is the use of Rydberg atoms to realize and explore the physics of strongly correlated spin systems, a direction that is also pursued by Lee et al. They evoke a scenario in which atoms are modeled by only two internal states, which is a huge simplification because an atom has infinitely many electronic levels. This two-level approximation is valid if the frequency of the laser used to excite Rydberg levels is closely resonant with only a single electronic transition. In this situation, the electronic ground state can be regarded as the down state and the Rydberg level as the up state of a pseudospin. Shifts in electronic levels caused by the presence of two or more Rydberg atoms then directly translate to a spin-spin interaction. The coupling to the excitation laser produces an effective magnetic field. Recent work has shown that these Rydberg pseudospin systems form a versatile toolbox for the study of critical phenomena [6], exotic quantum phases [7, dynamical crystallization [8], order-disorder phase transitions [9], as well as the thermalization of closed quantum systems [10].

Lee et al. include a final but important ingredient- dissipation. Like all excited states, Rydberg states are prone to spontaneous emission of photons. This 
a
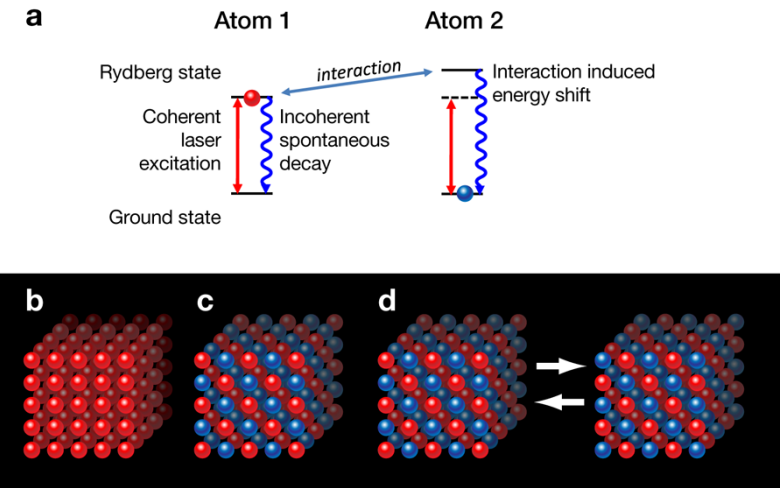

FIG. 1: (a) The rich dynamical behavior of the atoms in the lattice is determined by a competition between the coherent excitation of Rydberg states, the interaction among excited atoms, and dissipation caused by radiative decay. (b)-(d) Exaggerated sketch of the three phases that can be assumed. The stationary fixed-point solutions of the nonlinear mean field equations either (b) exhibit a uniform density of Rydberg atoms or (c) a checkered density distribution that breaks the sublattice symmetry. (d) A Hopf bifurcation can lead to an instability that gives rise to an oscillatory steady-state solution in which the density difference between the two sublattices oscillates in time. (APS/Alan Stonebraker)

aspect is often disregarded, as the lifetime of Rydberg states can be about $100 \mu \mathrm{s}$, longer than the typical duration of an experiment. Here, however, dissipation is rendered into a feature rather than a problem, which together with the involved coherent processes, i.e., the laser excitation and the interaction between Rydberg atoms [see Fig. 1(a)], produces an intricate dynamical behavior.

Mathematically, the dynamics of this open spin system is governed by a Lindblad master equation. This equation captures the coherent quantum mechanical evolution and at the same time permits the inclusion of incoherent processes. Lee et al. solve it with a mean-field approach, where each spin experiences a fictitious averaged interaction potential - the mean-field - produced by the spins on the remaining lattice sites. In a first attempt, all lattices sites are assumed to be equivalent. This ansatz leads to a system of coupled equations that are similar to the optical Bloch equations but contain a nonlinearity due to the Rydberg-Rydberg interaction. Lee et al. perform an analysis of the steady state of these equations. Such a steady state always exists but here turns out to be unstable for certain combinations of the laser parameters and the interaction strength. A closer look reveals that, in particular, perturbations with a wavelength twice the lattice spacing trigger these instabilities.

This decisive hint guides the authors to an augmented mean-field ansatz in which they break the system into two sublattices, each of which is described by its own mean field. Subsequent analysis of the resulting coupled equations reveals the existence of two stable fixed points, i.e., time-independent, steady-state solutions. One type of fixed point corresponds to a spatially uniform excitation of Rydberg atoms, while the other one shows an unequal population of the two sublattices. The broken sublattice symmetry of the system is reminiscent of an antiferromagnetic state.

Lee and his colleagues continue by exploring the structure of the fixed points as a function of the laser detuning parameter $\Delta$, which tells one how far off resonance the excitation laser frequency is with respect to the electronic transition from the ground state to the Rydberg state. For very large and negative $\Delta$, the nonlinear equations have just one fixed point that corresponds to a uniform density. This uniform fixed point becomes unstable with increasing $\Delta$ and stable nonuniform fixed points emerge from it. In an experiment, this should become visible in a continuous transition from a uniform density to an unequal occupation of the two sublattices.

In their numerical treatment, Lee et al. also observe that the nonuniform fixed points can become unstable via what is known in the theory of nonlinear dynamical systems as a Hopf bifurcation. Here the system undergoes a transition to a stable limit cycle in which the population of the two sublattices oscillates periodically in time. In total, the system therefore exhibits three distinct phases [see the densities sketched in Fig. 11(b)-(d)]: a uniform phase, a nonuniform or antiferromagnetic phase, and an oscillatory phase.

These findings provide a glimpse of the rich physics that should be accessible with Rydberg states of ultracold atoms. Experiments are about to catch up with the type of theoretical advance outlined in this paper; recently a first experimental implementation of a spin lattice system with Rydberg atoms was achieved [11.

\section{References}

[1] I. Bloch, J. Dalibard, and W. Zwerger, Rev. Mod. Phys. 80, 885 (2008).

[2] S. Diehl, A. Tomadin, A. Micheli, R. Fazio, and P. Zoller, Phys. Rev. Lett. 105, 015702 (2010).

[3] T. Lee, H. Häffner, and M. Cross, Phys. Rev. A 84, 031402 (2011).

[4] T. Gallagher, Rydberg Atoms (Cambridge University Press, Cambridge, 1984).

[5] M. Saffman, T. G. Walker, and K. Mølmer, Rev. Mod. Phys. 82, 2313 (2010); M. D. Lukin, M. Fleischhauer, R. Côté, L. M. Duan, D. Jaksch, J. I. Cirac, and P. Zoller, Phys. Rev. Lett. 87, 037901 (2001).

[6] H. Weimer, R. Löw, T. Pfau, and H. P. Büchler, Phys. Rev. Lett. 101, 250601 (2008).

[7] H. Weimer, M. Müller, I. Lesanovsky, P. Zoller, and H. P. Büchler, Nature Phys. 6, 382 (2010).

[8] T. Pohl, E. Demler, and M. D. Lukin, Phys. Rev. Lett. 104, $043002(2010)$.

[9] S. Ji, C. Ates, and I. Lesanovsky, Phys. Rev. Lett. 107, 060406 (2011); E. Sela, M. Punk, and M. Garst, Phys. Rev. B 84, 085434 (2011).

[10] I. Lesanovsky, B. Olmos, and J. P. Garrahan, Phys. Rev. Lett. 105, 100603 (2010).

(C) 2011 American Physical Society 
[11] M. Viteau, M. G. Bason, J. Radogostowicz, N. Malossi, D.

Ciampini, O. Morsch, and E. Arimondo, Phys. Rev. Lett. 107,

$060402(2011)$.

\section{About the Author}

\section{Igor Lesanovsky}

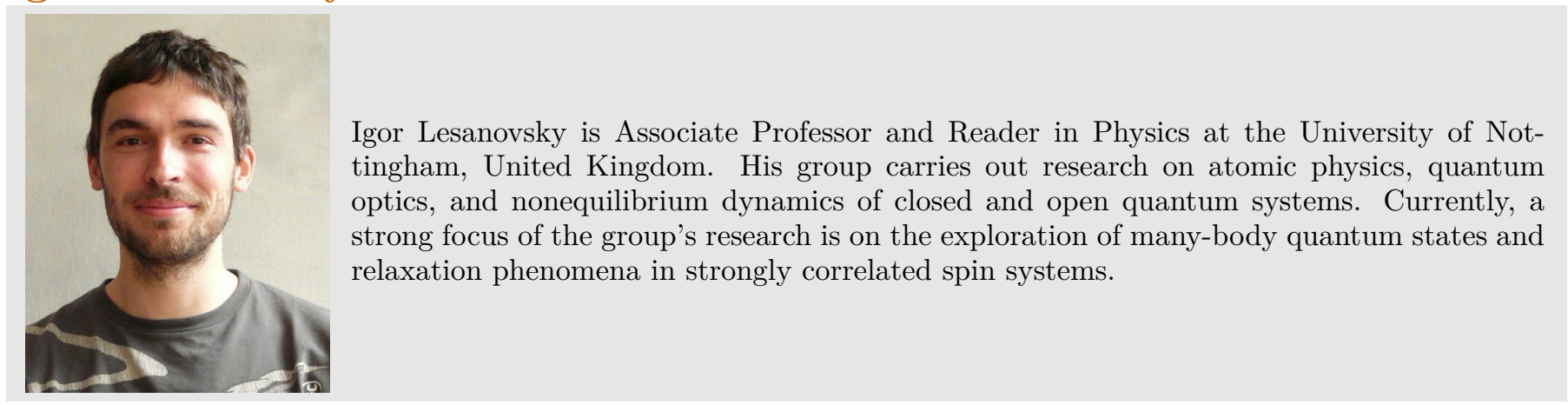

